\title{
Velocity-map imaging study of the photodissociation of acetaldehyde
}

\author{
H. A. Cruse and T. P. Softley \\ Department of Chemistry, University of Oxford, Chemistry Research Laboratory, Mansfield Road, Oxford \\ OX1 3TA, United Kingdom
}

(Received 12 November 2004; accepted 4 January 2005; published online 25 March 2005)

\begin{abstract}
Velocity-map imaging studies are reported for the photodissociation of acetaldehyde over a range of photolysis wavelengths $(317.5-282.5 \mathrm{~nm})$. Images are obtained for both the $\mathrm{HCO}$ and $\mathrm{CH}_{3}$ fragments. The mean rotational energy of both fragments increases with photodissociation energy, with a lesser degree of excitation in the $\mathrm{CH}_{3}$ fragment. The $\mathrm{CH}_{3}$ images demonstrate that the $\mathrm{CH}_{3}$ fragments are rotationally aligned with respect to the recoil direction and this is interpreted, and well modeled, on the basis of a propensity for forming $\mathrm{CH}_{3}$ fragments with $M \sim K$, where $M$ is the projection of the rotational angular momentum along the recoil direction. The origin of the $\mathrm{CH}_{3}$ rotation is conserved motion from the torsional and methyl-rocking modes of the parent molecule. Nonstatistical vibrational distributions for the $\mathrm{CH}_{3}$ fragment are obtained at higher energies. (C) 2005 American Institute of Physics. [DOI: 10.1063/1.1861886]
\end{abstract}

\section{INTRODUCTION}

In this paper we report on measurements of the product energy distributions for the dissociation of $\mathrm{CH}_{3} \mathrm{CHO}$ into $\mathrm{CH}_{3}$ and $\mathrm{HCO}$, at a range of photolysis wavelengths (317.5$282.5 \mathrm{~nm}$ ), using ion imaging of both fragments. The data presented here serve as a test of the validity of models that have been previously proposed to interpret the product energy distributions arising from this photodissociation process. In the present work, information has also been obtained on the alignment of the rotational angular momentum of the methyl fragments; these results support the interpretation of the $\mathrm{CH}_{3}$ rotational energy distribution data.

The potential energy surfaces of the lowest electronic states of acetaldehyde are represented schematically in Fig. 1. The main feature in the $\mathrm{CH}_{3} \mathrm{CHO}$ absorption spectrum is located between 250 and $350 \mathrm{~nm}$ and corresponds to the $n$ $\rightarrow \pi^{*}$ transition to the $S_{1}$ state. At excitation wavelengths shorter than $320.5 \mathrm{~nm}$ acetaldehyde dissociates to form $\mathrm{CH}_{3}+\mathrm{HCO}$. Experimental ${ }^{1-3}$ and theoretical ${ }^{4}$ studies indicate that these products correlate with the triplet surface of $\mathrm{CH}_{3} \mathrm{CHO}$. Therefore, the dissociation mechanism involves an intersystem crossing from the $S_{1}$ potential energy surface to the lowest triplet surface $T_{1}$ on which the dissociation proceeds over a small exit barrier. The intersystem crossing occurs on $\mathrm{a} \sim 10^{-8} \mathrm{~s}$ time scale, resulting in a dissociation that is slow overall compared to rotation of the parent molecule.

HCO rotational and vibrational energy distributions have been previously determined at a range of photolysis energies using laser-induced fluorescence (LIF) to detect the HCO fragment ${ }^{1-3,5}$. In the absence of a complete potential energy surface for the dissociation, statistical ${ }^{6}$ and impulsive ${ }^{7}$ models have been used to interpret this data and to infer information about the energy disposal in the $\mathrm{CH}_{3}$ cofragment. Most notably, no rotational excitation of $\mathrm{CH}_{3}$ is predicted from this work. ${ }^{2}$

Acetaldehyde is in many ways an interesting model sys- tem for photodissociation studies. The existence of the barrier in the exit channel poses a challenge to understanding the dynamics. Furthermore, the dissociation to form two polyatomic fragments raises important questions about the correlation between the vibration-rotation distributions of the two fragments, i.e., which quantum states of one fragment are formed in conjunction with a specific quantum state of the other fragment.

\section{EXPERIMENT}

\section{A. Description of the apparatus}

The photodissociation of acetaldehyde is studied in this work using the velocity-map imaging technique ${ }^{8}$ and a tunable photodissociation source. The nascent $\mathrm{CH}_{3}$ or $\mathrm{HCO}$ fragments are ionized using resonance-enhanced multiphoton

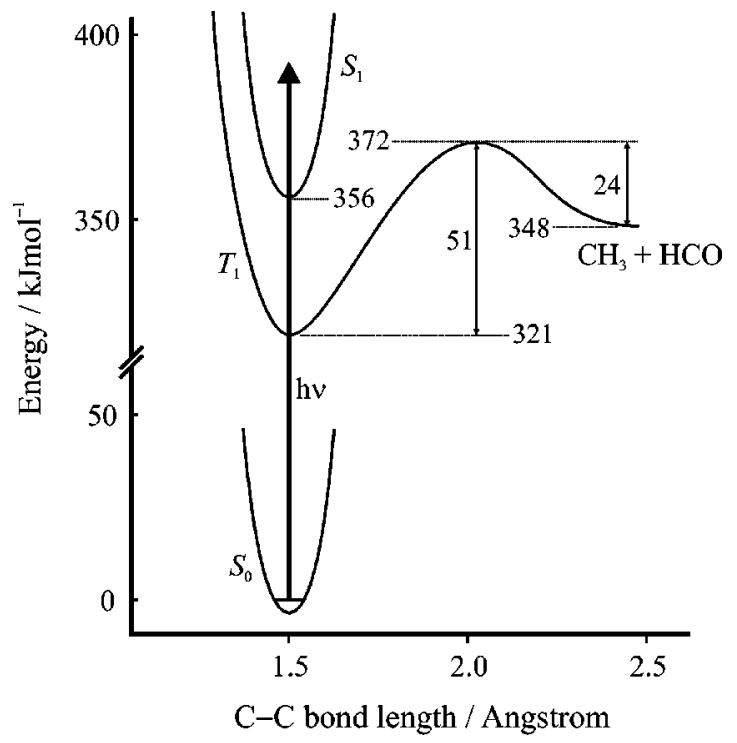

FIG. 1. Schematic potential energy diagram for the electronic states of acetaldehyde relevant to photodissociation in the range 250-350 nm (adapted from Ref. 2). All energies are given in $\mathrm{kJ} \mathrm{mol}^{-1}$. 


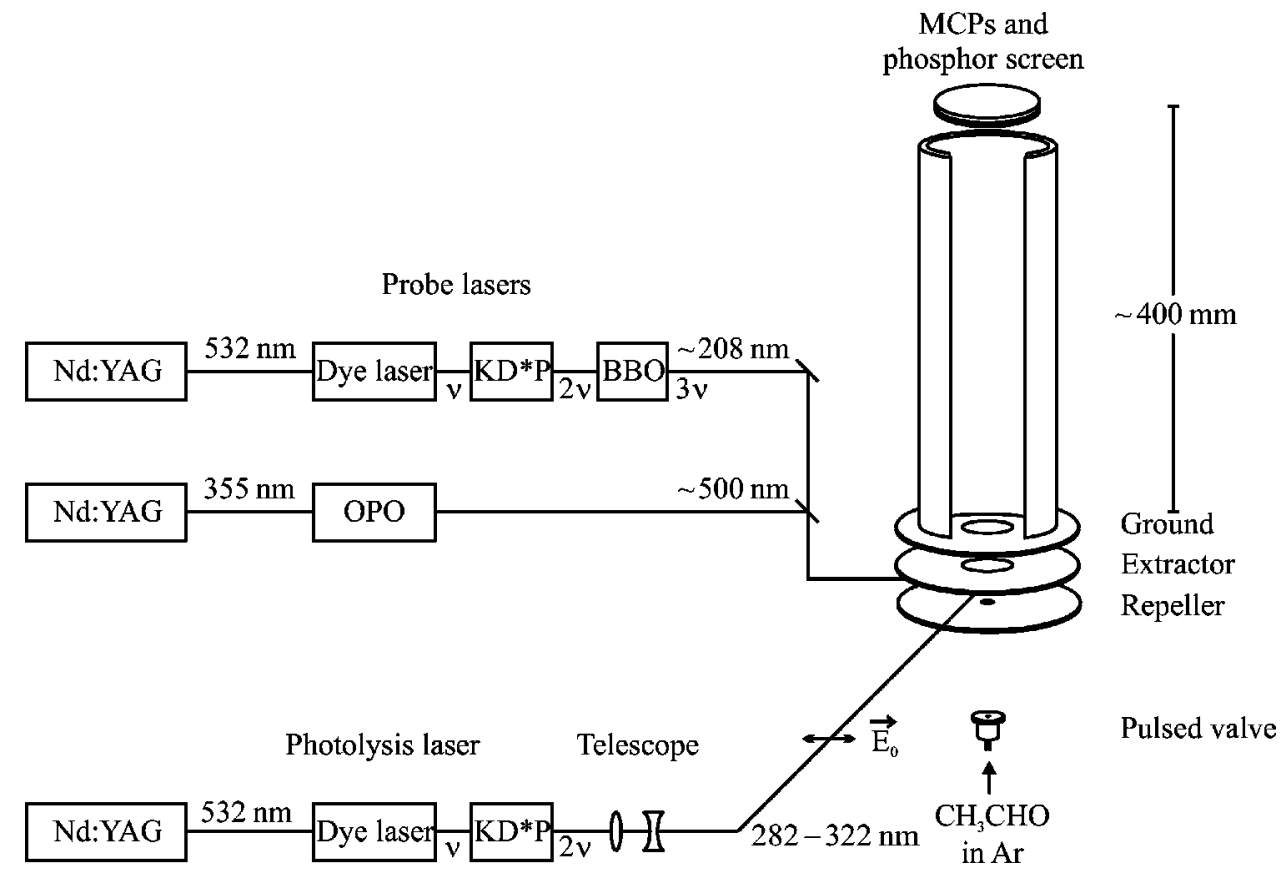

FIG. 2. Schematic diagram of the experimental layout used for the ionimaging studies on the photodissociation of $\mathrm{CH}_{3} \mathrm{CHO}$ (and calibration using $\left.\mathrm{CH}_{3} \mathrm{I}\right)$. The laser setup shown is that used for the $\left(1+1^{\prime}\right)$ REMPI detection of $\mathrm{HCO}$. ionization (REMPI) with a short time delay between the photodissociation and probe lasers. A schematic of the experimental apparatus is shown in Fig. 2. A supersonic free-jet expansion of acetaldehyde (14\% in argon, backing pressure 2.5 bars) produced by a pulsed nozzle (General Valve, $1 \mathrm{~mm}$ orifice, $400 \mu$ s pulse duration, $10 \mathrm{~Hz}$ repetition rate) is intersected by the frequency-doubled output, $\lambda=282-320 \mathrm{~nm}$, of a Nd:YAG pumped dye laser (Spectron SL4000G, $532 \mathrm{~nm}$ output/SL803). The UV power of this photolysis laser is typically $\sim 2 \mathrm{~mJ}$ per pulse, and before being introduced into the chamber the beam is reduced to $\sim 5 \mathrm{~mm}$ in diameter using a telescope.

A two-color $\left(1+1^{\prime}\right)$ REMPI detection scheme is employed to detect the HCO fragments. The frequency-tripled output of a second Nd:YAG pumped dye laser (Spectron SL4000G, $532 \mathrm{~nm}$ output/SL803) is used to excite ground state $\mathrm{HCO}$ to the $3 p^{2} \Pi\left(03^{0} 0\right) K=0\left(\Sigma^{-}\right)$intermediate state (see Sec. III) at $208.4 \mathrm{~nm}$, while the second color is provided by a Nd:YAG pumped optical parametric oscillator (Spectra Physics GCR 190, 355 nm output, with MOPO 730, 10 mJ per pulse) operating at around $500 \mathrm{~nm}$. The use of a highpower visible laser for the second-photon step to the nearthreshold continuum affords dramatic signal enhancement relative to one-color $(1+1)$ REMPI detection. ${ }^{9}$ The two probe lasers are combined on a dichroic mirror and are introduced into the chamber collinearly through a $f=20 \mathrm{~cm}$ fused silica lens, in a direction perpendicular to the photolysis laser.

For ion-imaging studies on the $\mathrm{CH}_{3}$ fragments, a onecolor $(2+1)$ REMPI scheme is employed, resonant with the origin band of the $3 p\left({ }^{2} A_{2}^{\prime \prime}\right) \leftarrow \tilde{X}\left({ }^{2} A_{2}^{\prime \prime}\right)$ transition. The frequency-doubled output of a dye laser at $\sim 333 \mathrm{~nm}$ (using a 4:1 DCM:Pyridine-1 dye mix) is used to excite this transition. The probe laser is tightly focused into the interaction region using a $f=20 \mathrm{~cm}$ fused silica lens.

In both the $\mathrm{CH}_{3}$ and $\mathrm{HCO}$ imaging studies, the polarization of the photolysis laser is chosen to be horizontal so as to lie in the plane of the detector, while the polarization of the first probe laser can be rotated from horizontal to vertical using a quartz half-wave plate. For both detection schemes the photolysis-probe delay is optimized at $\sim 10 \mathrm{~ns}$. Laser wavelengths are calibrated using a wavemeter (Burleigh WA5500).

The velocity-mapping ion lens is slightly modified from the original design of Eppink and Parker $^{8}$ and consists of three stainless steel electrodes of $140 \mathrm{~mm}$ diameter and 1.0 $\mathrm{mm}$ thickness, spaced at $15 \mathrm{~mm}$ intervals. The extractor and ground electrodes have apertures of diameter $15 \mathrm{~mm}$ and the supersonic free-jet expansion enters the ion lens through an opening of $2 \mathrm{~mm}$ diameter in the repeller plate, which is situated $\approx 45 \mathrm{~mm}$ downstream from the pulsed valve orifice. The lasers intersect the jet midway between the repeller and extractor plates. The experimental chamber is maintained at a pressure of $1 \times 10^{-5}$ mbars during signal acquisition.

In common with other velocity-map imaging setups, all trajectories with the same initial velocity are mapped onto the same position within the focal plane. For the $400 \mathrm{~mm}$ field-free drift region of this experiment, optimal focusing is attained for $V_{\mathrm{E}} / V_{\mathrm{R}}=0.68$, where $V_{\mathrm{E}}$ and $V_{\mathrm{R}}$ are the potentials applied to the extractor and repeller plates, respectively. A typical value of $V_{\mathrm{R}}=2 \mathrm{kV}$ is employed in the acetaldehyde experiments. The field applied in the extraction region also serves to accelerate the ions towards the detector and to compress the fragment cloud in the time-of-flight dimension.

The detector consists of a dual microchannel plate (MCP)/phosphor screen assembly (Gallileo, $40 \mathrm{~mm}$ diameter). Mass selectivity is achieved by using a fast highvoltage switch (Behlke, $10 \mathrm{~ns}$ rise time) to pulse the front MCP from ground to $-200 \mathrm{~V}$ at the arrival time of the species of interest. Rapid pulsing of the detector ensures minimal distortion of the ion trajectories by the field surrounding the front MCP. REMPI spectra are recorded using a photomultiplier tube (PMT) to collect the fluorescence from the 


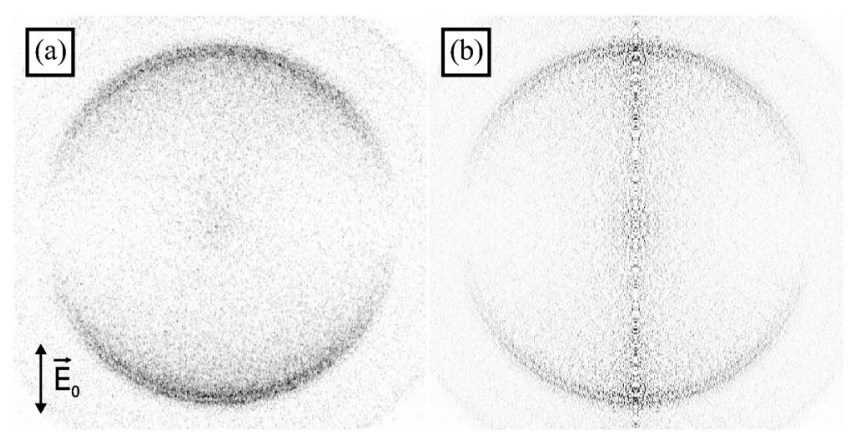

FIG. 3. (a) Raw $\mathrm{CH}_{3}^{+}$ion image obtained from the photodissociation of $\mathrm{CH}_{3} \mathrm{I}$ at $296.02 \mathrm{~nm}\left(33781 \mathrm{~cm}^{-1}\right)$ and acquired over 3000 laser shots. The $\mathrm{CH}_{3}$ fragment was probed via the $Q$ branch of the $3 p\left({ }^{2} A_{2}^{\prime \prime}\right) \leftarrow \tilde{X}\left({ }^{2} A_{2}^{\prime \prime}\right)$ origin band. (b) Image reconstructed using the BASEX Abel inversion package. The polarization direction of the photolysis laser is indicated in panel (a).

phosphor screen. The PMT output is amplified, averaged over 10 shots by a boxcar (Stanford Research Systems, SR250) and recorded by a PC.

Position-sensitive information is recorded using a freerunning charge-coupled device $(\mathrm{CCD})$ camera (Proxitronic HR0, $768 \times 576$ pixels, $50 \mathrm{~Hz}$ repetition rate) and sent to a frame grabber (Coreco Ultra II). Image acquisition is controlled by a PC using VISILOG 5.1 software (Noesis) and the data are averaged over a cycle of 20 frames. Typically, each image consists of 300 acquisition cycles. The threedimensional (3D) velocity distribution is reconstructed from the raw images using the BASEX Abel inversion routine ${ }^{10}$ (program supplied by Dribinski and Reisler).

\section{B. Calibration of the velocity-mapping setup}

In this work the instrumental resolution and the magnification factor of the ion lens were determined using the photodissociation of $\mathrm{CH}_{3} \mathrm{I}$ as a calibration system. Figure 3(a) shows a raw ion image of $\mathrm{CH}_{3}$ formed by the photodissociation of $\mathrm{CH}_{3} \mathrm{I}$ at a vacuum wavelength of $296.02 \mathrm{~nm}$ (a wave number of $\left.33781 \mathrm{~cm}^{-1}\right) . \mathrm{CH}_{3}$ was probed using $(2+1)$ REMPI excitation via the $Q$ branch of the $3 p\left({ }^{2} A_{2}^{\prime \prime}\right)$ $\leftarrow \tilde{X}\left({ }^{2} A_{2}^{\prime \prime}\right)$ transition as described in Sec. II A. The BASEX Abel inversion package ${ }^{10}$ was used to reconstruct the central slice through the full 3D photofragment distribution, shown in Fig. 3(b), from which the fragment kinetic-energy distribution shown in Fig. 4 was derived. All the peaks in the figure correspond to the formation of $\mathrm{I}^{*}$ and ground-state $\mathrm{CH}_{3}$. There is significant population of the low frequency vibrational modes of $\mathrm{CH}_{3} \mathrm{I}$ in the supersonic expansion and the higher fragment kinetic-energy peaks result from photodissociation of vibrationally excited $\mathrm{CH}_{3} \mathrm{I}$ with one or two quanta of energy in the $\nu_{3} \mathrm{C}-\mathrm{I}$ stretching mode.

The kinetic-energy distribution was simulated following the procedure of Eppink and Parker. ${ }^{11}$ The population of each $\mathrm{CH}_{3}$ rotational state $(N, K)$, as determined in Ref. 12 , is displayed in the inset of Fig. 4 at the corresponding kinetic energy, $E_{\text {kin }}^{\mathrm{CH}_{3}}(N, K)$. The rotational distributions were assumed to be independent of the initial parent vibrational energy. These populations were convoluted with a Gaussian broadening function of full width at half maximum (FWHM) $200 \mathrm{~cm}^{-1}$ to produce the example simulation shown in Fig.

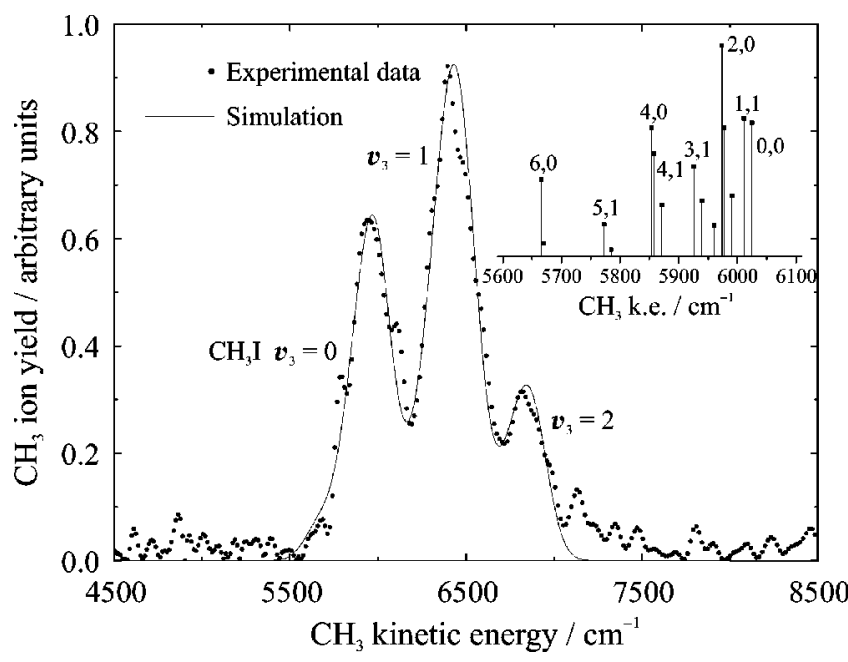

FIG. 4. The experimental kinetic-energy distribution for the $\mathrm{CH}_{3}$ fragment, derived from the Abel-inverted image shown in Fig. 3(b), and the simulation calculated using the procedure described in Sec. II B. The peaks due to population of different vibrational levels of the parent $\mathrm{CH}_{3} \mathrm{I} \nu_{3}$ mode are labeled in the figure. The inset shows the population of $\mathrm{CH}_{3}$ vibrationrotation states $(N, K)$ arising from the photodissociation of $\mathrm{CH}_{3} \mathrm{I}\left(v_{3}=0\right)$, as determined in Ref. 12. The $\mathrm{CH}_{3}$ kinetic energies are determined from the energies of the $(N, K)$ states by conservation of energy.

4. The broadening is consistent with the predicted maximum resolution for the velocity-mapping apparatus used, assuming that a block of $4 \times 4$ pixels is illuminated on the CCD camera by each ion event. ${ }^{11}$ The calibration of the ion lens magnification was performed by stretching the experimental kinetic-energy distribution to best fit the simulated data.

\section{HCO SPECTROSCOPY AND DETECTION}

The ${ }^{2} A^{\prime}$ ground state of $\mathrm{HCO}$ has an equilibrium angle of $\approx 120^{\circ}$ but can be described as a pseudosymmetric top with rotational energy levels given by

$$
\begin{aligned}
F^{\prime \prime}(N, K)= & \bar{B}^{\prime \prime} N(N+1)+\left(A^{\prime \prime}-\bar{B}^{\prime \prime}\right) K^{2}-D_{N}^{\prime \prime} N^{2}(N+1)^{2} \\
& -D_{N K}^{\prime \prime} N(N+1) K^{2}-D_{K}^{\prime \prime} K^{4},
\end{aligned}
$$

where $\bar{B}^{\prime \prime}=1 / 2\left(B^{\prime \prime}+C^{\prime \prime}\right)$. $A^{\prime \prime}, B^{\prime \prime}$, and $C^{\prime \prime}$ denote the rotational constants and $D_{N}^{\prime \prime}, D_{N K}^{\prime \prime}$, and $D_{K}^{\prime \prime}$ are the centrifugal distortion constants. Each $F^{\prime \prime}(N, K)$ level is split by the asymmetry into two sublevels. In the present work only the $K$ $=1$ levels are studied and the splitting of the $F_{c}$ and $F_{d}$ sublevels for $K=1$ is well approximated by the expression ${ }^{13,14}$

$$
F_{d}(N, 1)-F_{c}(N, 1)=\frac{1}{2}(B-C) N(N+1) \text {. }
$$

The $3 p^{2} \Pi\left(A^{\prime \prime}\right)$ state is the lowest sharp Rydberg state of HCO. It has a linear configuration and its geometry is very close to that of the $\mathrm{HCO}^{+}$cation ground state. For the present purposes the spin doubling is neglected and the rotational term values are given by the standard expression

$$
F^{\prime}(N)=B^{\prime} N(N+1)-D^{\prime} N^{2}(N+1)^{2} \text {. }
$$

The REMPI spectrum of $\mathrm{HCO}$ recorded via the $3 p^{2} \Pi\left(A^{\prime \prime}\right)$ state consists of a long progression in the $\nu_{2}$ bending mode. Each band is divided into subbands according to the initial and final values of $K$. For the linear $3 p^{2} \Pi\left(A^{\prime \prime}\right)$ state, which displays the Renner-Teller effect, $K$ is the re- 


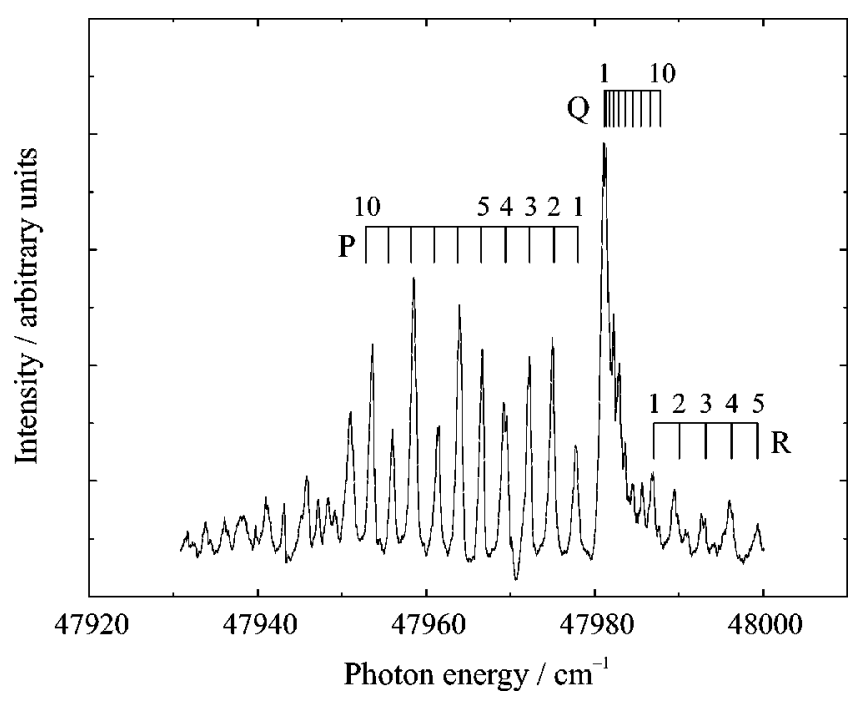

FIG. 5. $\left(1+1^{\prime}\right)$ REMPI spectrum of the $K^{\prime}=0\left(\Sigma^{-}\right) \leftarrow K^{\prime \prime}=1(\Pi)$ subband of the HCO $3 p^{2} \Pi A^{\prime \prime}(030) \leftarrow \widetilde{X}^{2} A^{\prime}(000)$ transition. The combs above the spectrum show the transition frequencies calculated using Eqs. (1)-(3).

sultant component of the vibrational and electronic angular momentum along the molecular axis. Figure 5 displays the $\left(1+1^{\prime}\right)$ REMPI spectrum of the $3 p^{2} \Pi A^{\prime \prime}(030) K^{\prime}=0\left(\Sigma^{-}\right)$ $\leftarrow \tilde{X}^{2} A^{\prime}(000) K^{\prime \prime}=1(\Pi)$ subband. This $\Delta K=-1$ subband has a perpendicular band structure, with clear $P, Q$, and $R$ branches. The transition frequencies calculated using Eqs. (1)-(3) and the rotational constants and vibrational term energies of Ref. 15 are represented by the combs above the spectrum in Fig. 5. The excellent agreement with the experimental spectrum confirms the assignment of this subband (which is identical to the assignment given in Ref. 15.)

\section{PHOTOFRAGMENT YIELD SPECTRA}

Photofragment excitation (PHOFEX) spectra for the dissociation of $\mathrm{CH}_{3} \mathrm{CHO}$ into $\mathrm{CH}_{3}+\mathrm{HCO}$ were recorded by detecting either $\mathrm{HCO}$ or $\mathrm{CH}_{3}$ by REMPI excitation as the photolysis laser was scanned. Figure 6 shows the PHOFEX spectrum of the HCO fragment, obtained by fixing the first probe laser on the peak of the $Q$ branch of the $3 p^{2} \Pi A^{\prime \prime}(030) K^{\prime}=0\left(\Sigma^{-}\right) \leftarrow \tilde{X}^{2} A^{\prime}(000) K^{\prime \prime}=1(\Pi) \quad$ subband displayed in Fig. 5. A section of the corresponding $\mathrm{CH}_{3}$ PHOFEX spectrum is superimposed and was obtained by tuning the probe laser to the $Q$ branch of the $3 p\left({ }^{2} A_{2}^{\prime \prime}\right)$ $\leftarrow \tilde{X}\left({ }^{2} A_{2}^{\prime \prime}\right)$ origin band of $\mathrm{CH}_{3}$.

As would be expected, the two PHOFEX spectra are very similar in appearance, and any differences between them can be attributed to the lower signal-to-noise ratio for $\mathrm{CH}_{3}$ detection. The spectra are in excellent agreement with the only previously published PHOFEX spectra, which were recorded by monitoring a transition in the LIF excitation spectrum of HCO. ${ }^{1,3,5}$ The onset of the PHOFEX signal occurs at a wavelength of $320.5 \mathrm{~nm}\left(\equiv 31200 \mathrm{~cm}^{-1}\right.$ or $373.2 \mathrm{~kJ} \mathrm{~mol}^{-1}$ ), which is consistent with the threshold for the $\mathrm{CH}_{3}+\mathrm{HCO}$ channel as calculated from the energy of the $T_{1}$ $\leftarrow S_{0}(0-0)$ transition $\left[321.5 \mathrm{~kJ} \mathrm{~mol}^{-1}\right.$ (Ref. 16)] and the the-

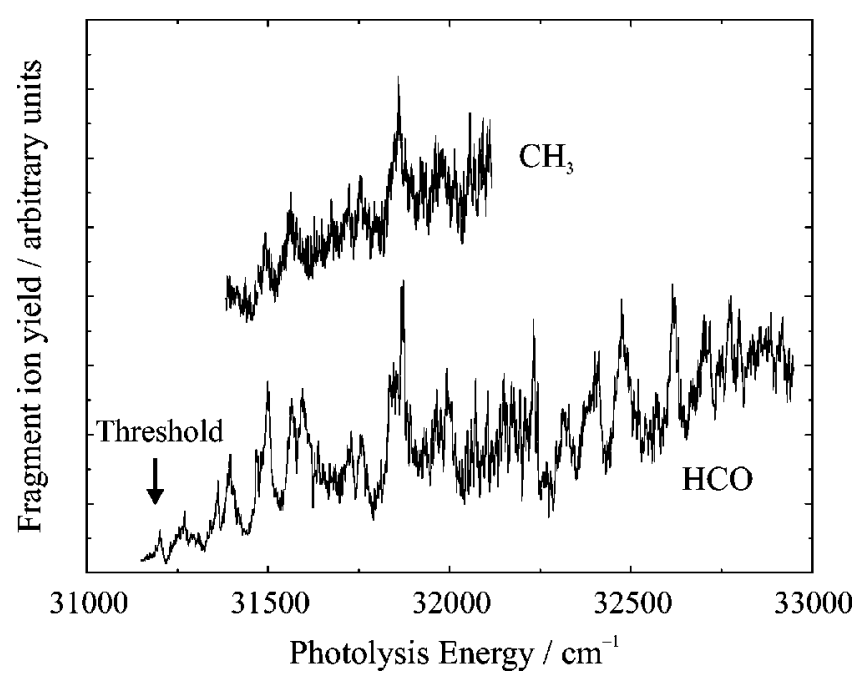

FIG. 6. PHOFEX spectra for the photodissociation of acetaldehyde, obtained by scanning the photodissociation laser while detecting the $\mathrm{HCO}$ or $\mathrm{CH}_{3}$ fragment using REMPI. The position of the dissociation threshold is indicated on the $\mathrm{HCO}$ spectrum.

oretical estimate of the barrier height on the $T_{1}$ surface [51.9 $\mathrm{kJ} \mathrm{mol}^{-1}$ (Refs. 4, 17, and 18)]. It is also consistent with previous LIF-PHOFEX measurements. ${ }^{5}$

The sharp features observed in the PHOFEX spectrum are a continuation of the vibronic structure seen in the fluorescence excitation spectrum of acetaldehyde, ${ }^{1,19-21}$ and were tentatively assigned by Hanazaki and co-workers ${ }^{1}$ as combination bands of the $\nu_{4}$ (CO stretch), $\nu_{14}$ (methyl-rocking), and $\nu_{15}\left(\mathrm{CH}_{3}\right.$ torsion $)$ vibrational modes of acetaldehyde. Consideration of the geometries of the $S_{0}$ and $S_{1}$ states reveals that these are the Franck-Condon active modes of the $S_{0} \rightarrow S_{1}$ excitation. $^{21}$

\section{IMAGE ANALYSIS}

Figure 7(a) shows a raw ion image of nascent $\mathrm{HCO}$ fragments formed from the photolysis of $\mathrm{CH}_{3} \mathrm{CHO}$ at a vacuum wavelength of $292 \mathrm{~nm}$, corresponding to an excitation energy that is $5156 \mathrm{~cm}^{-1}$ above the barrierless threshold for the formation of ground state $\mathrm{HCO}+\mathrm{CH}_{3}$ (or $3150 \mathrm{~cm}^{-1}$ above the calculated barrier height). $\mathrm{HCO}$ was probed using the
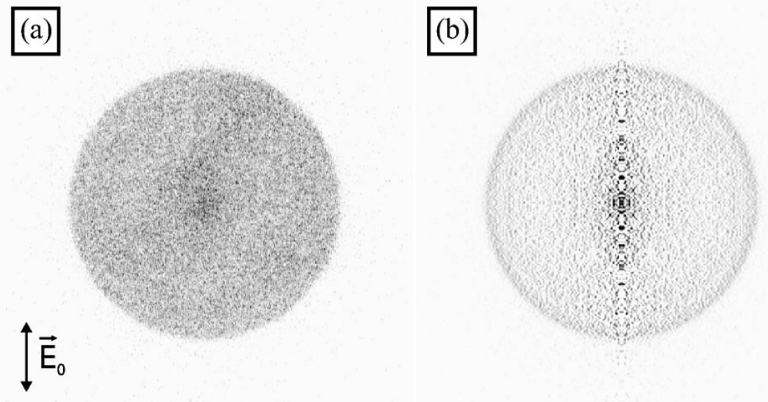

FIG. 7. (a) Raw ion image of the nascent $\mathrm{HCO}$ fragments from the photodissociation of $\mathrm{CH}_{3} \mathrm{CHO}$ at $292.00 \mathrm{~nm}\left(34247 \mathrm{~cm}^{-1}\right)$, acquired over 8000 laser shots. The $\mathrm{HCO}^{+}$ion intensity in the center of the image arises from fragmentation of the $\mathrm{CH}_{3} \mathrm{CHO}^{+}$ions that are produced by nonresonant ionization of the parent molecules. (b) Image reconstructed using the BASEX Abel inversion routine. 


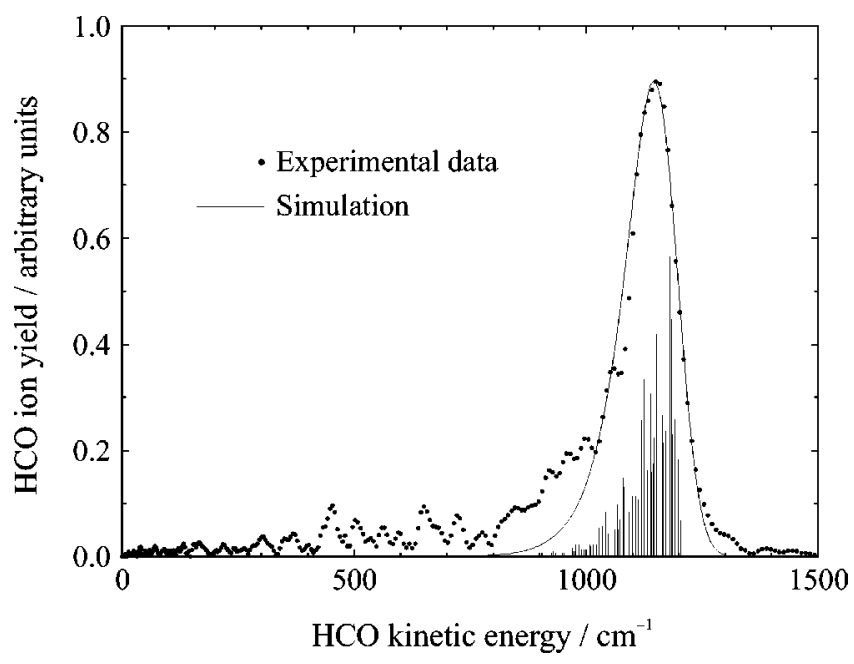

FIG. 8. Kinetic-energy profile of HCO fragments, determined from the reconstructed image obtained at a photodissociation energy $3529 \mathrm{~cm}^{-1}$ above the barrierless threshold. The stick diagram shows the underlying $\mathrm{CH}_{3}$ rotational energy distribution used to obtain the simulation.

$\left(1+1^{\prime}\right)$ REMPI scheme via the $Q$ branch of the $3 p^{2} \Pi A^{\prime \prime}(030) K^{\prime}=0\left(\Sigma^{-}\right) \leftarrow \tilde{X}^{2} A^{\prime}(000) K^{\prime \prime}=1(\Pi) \quad$ subband (see Sec. III). As discussed earlier, the dissociation is slow with respect to the parent rotational period and as a result the image is essentially isotropic. The central slice of this distribution obtained from the Abel inversion is displayed in Fig. 7(b). It should be noted that the geometry used in the present experiments is such that the photodissociation laser and probe laser polarizations cannot be simultaneously parallel to each other and to the face of the detector. Thus our results do not prove that the distribution is isotropic, but this can be inferred from the previous lifetime data. The normalized radial distribution is obtained by integration of the appropriately weighted image over the polar angle $\theta$. Using the velocity-mapping calibration factor determined from $\mathrm{CH}_{3} \mathrm{I}$ photolysis, the kinetic-energy-release curve, and hence the product rotational and vibrational distributions, can be calculated.

Images of the $\mathrm{HCO}$ product were recorded at photodissociation energies covering the range $2405 \mathrm{~cm}^{-1}-6307 \mathrm{~cm}^{-1}$ above the barrierless threshold for the formation of $\mathrm{HCO}$ $+\mathrm{CH}_{3}$ (or $\approx 400 \mathrm{~cm}^{-1}-4300 \mathrm{~cm}^{-1}$ above the top of the barrier.) In each case product rotational and vibrational distributions were calculated by comparison of the experimental and simulated kinetic-energy-release curves.

\section{A. Product rotational energy distributions 1. $\mathrm{CH}_{3}$ rotational energy distributions from $\mathrm{HCO}$ images}

An illustrative $\mathrm{HCO}$ kinetic-energy distribution, recorded $3529 \mathrm{~cm}^{-1}$ above the barrierless threshold, is shown in Fig. 8. Neglecting the internal energy of the parent, the $\mathrm{HCO}$ fragment kinetic energy $E_{\mathrm{kin}}^{\mathrm{HCO}}(N, K)$ is given by

$$
E_{\text {kin }}^{\mathrm{HCO}}(N, K)=\frac{m_{\mathrm{CH}_{3}}}{m_{\mathrm{CH}_{3} \mathrm{CHO}}}\left[h \nu-D_{0}-E_{\mathrm{HCO}}-F(N, K)\right],
$$

where $\nu$ is the frequency of the photolysis laser, $F(N, K)$ is the term energy for the $\mathrm{CH}_{3}$ rotational level, and $E_{\mathrm{HCO}}$ is the internal energy of the HCO fragment, which is well defined by the REMPI detection scheme. $D_{0}$ is the dissociation energy of ground state $\mathrm{CH}_{3} \mathrm{CHO}$ into ground state fragments, $\mathrm{CH}_{3}+\mathrm{HCO}$, which is taken to be $349.6 \mathrm{~kJ} \mathrm{~mol}^{-1} \cdot{ }^{22}$ At the probe laser wave number used in these experiments, $47982.3 \mathrm{~cm}^{-1}$, the HCO absorption is centered on the $Q(4)$ line with some excitation of the adjacent $Q(3)$ and $Q(5)$ lines under the $0.35 \mathrm{~cm}^{-1}$ laser bandwidth. However, the simulated kinetic-energy-release profile shown in Fig. 8 is insensitive to the inclusion of the contribution from these other lines. The $Q(4)$ line originates from the $\tilde{X}^{2} A^{\prime}(4,1)$ level of the ground state, which corresponds to an HCO internal energy $E_{\mathrm{HCO}}$ of $28.6 \mathrm{~cm}^{-1}$. Since $E_{\mathrm{HCO}}$ is fixed, the width of the peak in the kinetic-energy profile is determined solely by the experimental broadening function and the rotational distribution of the $\mathrm{CH}_{3}$ cofragment. To simulate the HCO kineticenergy distribution, the term energy, $F(N, K)$, of each energetically accessible $\mathrm{CH}_{3}$ rotational state was calculated using the standard symmetric top formula and the rotational constants given in Ref. 23.

The contributions of the individual $\mathrm{CH}_{3}$ rotational levels to the kinetic-energy distribution are not resolved, and therefore a precise determination of the form of the $\mathrm{CH}_{3}$ rotational population distribution is not possible. Although there is no $a$ priori reason to suppose that the distribution obeys Boltzmann statistics, a Boltzmann population distribution of the $\mathrm{CH}_{3}$ rotational states (multiplied by the nuclear spin statistical weight) is used to calculate the contribution of each $N, K$ state in the simulation of the HCO kinetic-energy distribution. The good fit between experiment and simulation suggests that this is a reasonable approximation to use for the form of the distribution in the absence of more detailed information. The effective temperature thus derived provides at least a relative measure of the disposal of energy into rotational degrees of freedom. It should be noted that the $\mathrm{CH}_{3}$ rotational distribution determined here is associated with the detection of the $\mathrm{HCO}$ cofragment in its $N=4, K=1$ level, and may not be identical to the total rotational distribution summed over all cofragment states.

The $\mathrm{CH}_{3}$ rotational state populations obtained for a Boltzmann rotational temperature of $200 \mathrm{~K}$ are shown in Fig. 8 at the corresponding values of $E_{\text {kin }}^{\mathrm{HCO}}(N, K)$, calculated assuming a photolysis laser frequency of $32619 \mathrm{~cm}^{-1}$. This discrete energy distribution is then convoluted with a Gaussian function, which is a measure of the instrumental resolution. Figure 8 displays the distribution obtained when a Gaussian of FWHM $91 \mathrm{~cm}^{-1}$ is used; this corresponds to a kinetic-energy resolution $\Delta E / E_{\text {trans }}$ of $7.5 \%$. The kinetic-energy-release curve shown in Fig. 8 was simulated by manually optimizing the $\mathrm{CH}_{3}$ rotational temperature and the Gaussian width until the best fit with the experimental data was obtained. The shape of the high-energy edge of the peak is determined predominantly by the width of the Gaussian function and 
TABLE I. Rotational excitation of the $\mathrm{CH}_{3}$ fragment. The available energy is measured with respect to the ground state products. The fitting procedure enables the temperature to be calculated to an accuracy of $\pm 10 \mathrm{~K}$.

\begin{tabular}{cccc}
\hline \hline $\begin{array}{c}\text { Excitation } \\
\text { energy } \\
\left(\mathrm{cm}^{-1}\right)\end{array}$ & $\begin{array}{c}\text { Available } \\
\text { energy } \\
\left(\mathrm{cm}^{-1}\right)\end{array}$ & $\begin{array}{c}\text { Rotational } \\
\text { temperature } \\
(\mathrm{K})\end{array}$ & $\begin{array}{c}\text { Rotational } \\
\text { energy } \\
\left(\mathrm{cm}^{-1}\right)\end{array}$ \\
\hline 31496 & 2405 & 100 & 104 \\
31857 & 2766 & 120 & 125 \\
32619 & 3529 & 200 & 208 \\
\hline \hline
\end{tabular}

was fitted accordingly. The low-energy edge, which corresponds to higher $\mathrm{CH}_{3}$ rotational excitation, was then fitted to obtain the Boltzmann temperature.

Table I displays the effective rotational temperatures $T_{\text {rot }}$ that were deduced in this procedure for $\mathrm{CH}_{3}$ fragments formed from the photodissociation of $\mathrm{CH}_{3} \mathrm{CHO}$ at a range of photolysis wavelengths. The corresponding mean rotational energies are also shown. Values are only displayed for the longer photolysis wavelengths at which no vibrational excitation of the methyl fragment is observed.

\section{HCO rotational distributions deduced from $\mathrm{CH}_{3}$ images}

Images of the methyl fragment can be analyzed in an analogous manner to obtain an estimate of the mean rotational energy of the $\mathrm{HCO}$ product. $\mathrm{CH}_{3}$ was detected using $(2+1)$ REMPI excitation via the $Q$ branch of the $3 p\left({ }^{2} A_{2}^{\prime \prime}\right)$ $\leftarrow X\left({ }^{2} A_{2}^{\prime \prime}\right)$ origin band. Individual rotational lines of this $Q$ branch are not resolved, and thus the simulation of the kinetic-energy curve must include a summation over all accessible $\mathrm{CH}_{3}$ rotational levels. For simplicity it is assumed that the $\mathrm{CH}_{3}$ fragments were ionized via the $Q$ branch to produce a signal proportional to the populations of the initial $\mathrm{CH}_{3}$ rotational states (these were determined in the fit of the $\mathrm{HCO}$ images to a Boltzmann distribution as discussed above.) Given the low rotational temperature of the $\mathrm{CH}_{3}$ fragment and the very $\operatorname{similar} B$ values in the ground and $3 p$ Rydberg states, the proportionality of the signal to the populations is expected to be a good approximation. The term energy $G(N, K)$ of each HCO rotational state was determined from Eq. (1) using the rotational constants given in Ref. 15. By analogy with Eq. (4), the kinetic energy imparted to the $\mathrm{CH}_{3}$ fragment, $E_{\mathrm{kin}}^{\mathrm{CH}_{3}}$, is given by

$$
\begin{aligned}
E_{\mathrm{kin}}^{\mathrm{CH}_{3}}= & \frac{m_{\mathrm{HCO}}}{m_{\mathrm{CH}_{3} \mathrm{CHO}}}\left[h \nu-D_{0}-F\left(N^{\mathrm{CH}_{3}}, K^{\mathrm{CH}_{3}}\right)\right. \\
& \left.-G\left(N^{\mathrm{HCO}}, K^{\mathrm{HCO}}\right)\right] .
\end{aligned}
$$

For reasons similar to those discussed in the analysis of the $\mathrm{CH}_{3}$ populations, a Boltzmann population distribution was also adopted for the HCO fragments. This is in accordance with earlier work by the groups of Hanazaki ${ }^{5}$ and Kable. ${ }^{2}$ However, it should be noted that in a later paper ${ }^{3}$ Lee and Chen reported that the rotational energy of HCO is preferentially partitioned along the $b$ and $c$ axes, rather than the inertial $a$ axis. This highlights the limitations of using the Boltzmann distribution and indicates that, as before, the Boltzmann temperatures derived here can only be regarded

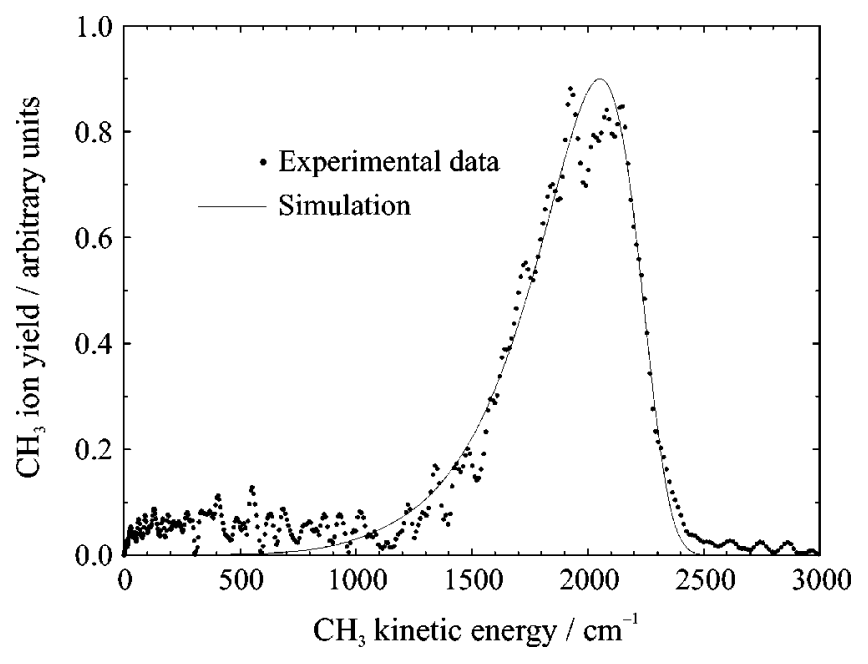

FIG. 9. Kinetic-energy distribution of $\mathrm{CH}_{3}$ fragments, determined from the reconstructed $\mathrm{CH}_{3}^{+}$ion image obtained at a photodissociation energy $3529 \mathrm{~cm}^{-1}$ above the barrierless threshold, with a simulated profile derived using the method described in the text.

as a crude measure of relative rotational excitation. As previously, the energy distribution was convoluted with a Gaussian function, and the HCO rotational temperature and FWHM of the Gaussian were optimized to best match the experimental data. By way of illustration, experimental and simulated kinetic-energy-release curves for $\mathrm{CH}_{3}$ fragments generated by the $32619 \mathrm{~cm}^{-1}$ photodissociation of $\mathrm{CH}_{3} \mathrm{CHO}$, $3529 \mathrm{~cm}^{-1}$ above the barrierless threshold for the formation of $\mathrm{CH}_{3}$ and $\mathrm{HCO}$, are displayed in Fig. 9. The effective rotational temperatures of the $\mathrm{HCO}$ fragment deduced from these measurements are shown in Table II for a series of photodissociation wavelengths, and it is immediately evident that the $\mathrm{HCO}$ product is formed with considerably greater rotational energy than the methyl cofragment. For both fragments the fraction of energy partitioned into rotation increases slightly with photolysis energy. The results obtained are consistent with previous estimates of the HCO rotational excitation determined by modeling the LIF spectrum of the $\widetilde{B}^{2} A^{\prime} \leftarrow \widetilde{X}^{2} A^{\prime}(0,0)$ transition of HCO. ${ }^{2,5}$

\section{Discussion of the rotational distributions}

As discussed previously, ${ }^{24,25}$ rotational excitation of fragments formed via a unimolecular photodissociation process may originate from either (a) rotational angular momentum of the parent being transferred to the fragments, (b) vibrational excitation of the parent, particularly of bendingtype modes, evolving into fragment rotational motion as a bond breaks, or (c) a torque imparted to the fragments as

TABLE II. Rotational excitation of the HCO product. The available energy is measured with respect to the ground state products.

\begin{tabular}{cccc}
\hline \hline $\begin{array}{c}\text { Excitation } \\
\text { energy } \\
\left(\mathrm{cm}^{-1}\right)\end{array}$ & $\begin{array}{c}\text { Available } \\
\text { energy } \\
\left(\mathrm{cm}^{-1}\right)\end{array}$ & $\begin{array}{c}\text { Rotational } \\
\text { temperature } \\
(\mathrm{K})\end{array}$ & $\begin{array}{c}\text { Rotational } \\
\text { energy } \\
\left(\mathrm{cm}^{-1}\right)\end{array}$ \\
\hline 31857 & 2766 & 300 & 313 \\
32619 & 3529 & 500 & 521 \\
32772 & 3681 & 550 & 573 \\
\hline
\end{tabular}




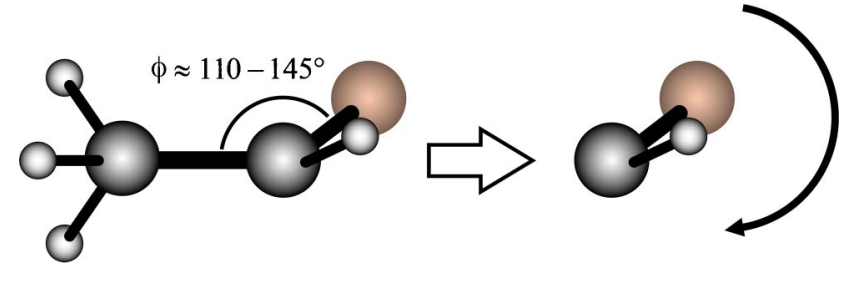

FIG. 10. Calculated transition state structure of acetaldehyde, illustrating one source of rotational angular momentum in the HCO fragment (adapted from Ref. 4).

they traverse the exit potential energy surface. Of these (a) is likely to be negligible in the present experiments in comparison with the other mechanisms since the rotational degrees of freedom of the parent are efficiently cooled in a supersonic expansion, and the rotational selection rules ensure that the rotational temperature does not change significantly on excitation to the predissociative $S_{1}$ state. The effect of exitchannel forces on product rotational excitation [mechanism (c)] has been estimated using the impulsive model, ${ }^{7,26}$ in which the $\mathrm{CH}_{3}$ and $\mathrm{HCO}$ fragments are treated as rigid rotors. Ab initio calculations of the transition state structure of $\mathrm{CH}_{3} \mathrm{CHO}$ at the top of the barrier on the $T_{1}$ potential energy surface ${ }^{4,27}$ indicate that the methyl group is bent out of the HCO plane by $35^{\circ}-70^{\circ}$, as depicted in Fig. 10. Therefore, when the $\mathrm{C}-\mathrm{C}$ bond is broken, the repulsive force along the direction of the $\mathrm{CH}_{3}$ symmetry axis is not directed so as to pass through the $\mathrm{HCO}$ center of mass, resulting in a torque about the $\mathrm{HCO}$ rotational axes and hence rotational excitation of this fragment. The nonplanar nature of the transition state leads to a preference for out-of-plane rotation of $\mathrm{HCO}$ rather than the in-plane boomerang motion characterized by $K_{a}=0$ (note that the $a$ axis lies close to the $\mathrm{CO}$ bond direction). Accordingly, the spectroscopic results of Kable and co-workers indicate a slight preference for $K_{a} \neq 0 .^{2}$ In contrast to this, no rotational excitation of the methyl radical is predicted by the impulsive model, since the force is directed through the $\mathrm{CH}_{3}$ center of mass and there is no torque. This simple description offers an explanation as to why $\mathrm{HCO}$ is formed with much greater average rotational excitation than $\mathrm{CH}_{3}$, and provides a useful starting point for a discussion of the product rotational energy distributions.

Table III lists the experimentally determined rotational energy of the HCO fragment (based on an assumed Boltzmann distribution), together with the rotational excitation predicted by the impulsive model, extrapolated from the calculations of Kable and co-workers. ${ }^{2}$ The agreement between

TABLE III. Rotational excitation of $\mathrm{HCO}$ as calculated by the impulsive model, in comparison with the experimental data.

\begin{tabular}{cccccc}
\hline \hline & \multicolumn{2}{c}{ Experimental data } & & \multicolumn{2}{c}{ Impulsive model } \\
\cline { 2 - 3 } \cline { 5 - 5 } $\begin{array}{c}\text { Available } \\
\text { energy } \\
\left(\mathrm{cm}^{-1}\right)\end{array}$ & $\begin{array}{c}\text { Rotational } \\
\text { energy of HCO } \\
\left(\mathrm{cm}^{-1}\right)\end{array}$ & $\begin{array}{c}\text { Fraction of } \\
\text { total energy }\end{array}$ & $\begin{array}{c}\text { Rotational } \\
\text { energy of HCO } \\
\left(\mathrm{cm}^{-1}\right)\end{array}$ & $\begin{array}{c}\text { Fraction of } \\
\text { total energy }\end{array}$ \\
\hline 2766 & 313 & 0.113 & & 497 & 0.180 \\
3529 & 521 & 0.148 & & 635 & 0.180 \\
3682 & 573 & 0.156 & 663 & 0.180 \\
\hline \hline
\end{tabular}

these values is reasonable, although in the impulsive model the fraction of energy imparted to rotation remains constant over all photodissociation wavelengths, and this is not observed experimentally. Furthermore, the model consistently overestimates the HCO rotational energy in the photolysis energy range considered here, while failing to account for the nonzero rotational energy of the $\mathrm{CH}_{3}$ fragment.

As discussed in Sec. IV, the $\nu_{15}$ torsional mode is strongly Franck-Condon active in the $S_{1} \leftarrow S_{0}$ transition of acetaldehyde. The $S_{1}$ and $T_{1}$ states of acetaldehyde have similar geometries, ${ }^{21,27}$ so it is reasonable to assume that any vibrational excitation remaining in the $\nu_{15}$ mode is conserved during the intersystem crossing. On dissociation the $\nu_{15}$ mode is partially converted to high- $K$ propeller-type rotation of the $\mathrm{CH}_{3}$ fragment about its $C_{3}$ axis, as indicated in Fig. 11, so this mechanism could account for the observed small degree of rotational excitation of the methyl radical. The $\nu_{15}$ vibrational mode will also induce additional rotational excitation in the HCO fragment.

The degree of vibrational excitation in the $S_{1}$ state of acetaldehyde will increase with photolysis energy, thus accounting for the observed increase in the energy partitioned into rotation at higher excitation energies. However, intramolecular vibrational redistribution (IVR) will disperse some of this energy amongst various modes in the $10^{-8} \mathrm{~s}$ before the intersystem crossing takes place and thus non-FranckCondon-active vibrational modes of acetaldehyde must also be considered. As discussed later (see Sec. VI), there is evidence that the $\nu_{14}$ methyl-rocking mode of the parent, which correlates adiabatically with a low- $K$ tumbling motion of the $\mathrm{CH}_{3}$ product [see Fig. 11(b)], also contributes to rotation of the $\mathrm{CH}_{3}$ fragment.

\section{B. Vibrational excitation of the methyl fragment}

At photodissociation energies in excess of $\sim 4000 \mathrm{~cm}^{-1}$ above the barrierless threshold for the formation of $\mathrm{CH}_{3}$ $+\mathrm{HCO}$, bands corresponding to the production of vibrationally excited $\mathrm{CH}_{3}$ are observed in the $\mathrm{HCO}$ ion images. As shown in Fig. 12, the degree of $\mathrm{CH}_{3}$ vibrational excitation increases with photolysis energy. These kinetic-energyrelease curves can be simulated by adapting the model described in Sec. V A to incorporate vibrational excitation of the $\mathrm{CH}_{3}$ fragment. This is achieved by using Eq. (4) to calculate the kinetic-energy release corresponding to the $F(N, K)$ levels for all accessible vibration-rotation states, the energies of which are determined using the rotational constants given in Ref. 23. The rotational temperature, the relative population of each vibrational level (measured as the integrated area under each vibrational peak), and the Gaussian instrumental width were adjusted manually to provide the best fit to the experimental data.

Experimental and simulated $\mathrm{HCO}$ kinetic-energy-release curves for the photodissociation of $\mathrm{CH}_{3} \mathrm{CHO}$ at an energy of $5156 \mathrm{~cm}^{-1}$ above the barrierless $\mathrm{CH}_{3}+\mathrm{HCO}$ threshold are shown in Fig. 13. As a result of the overlap of some vibrational bands it was not possible to fully decouple the effects of the Gaussian broadening and the rotational population dis- 

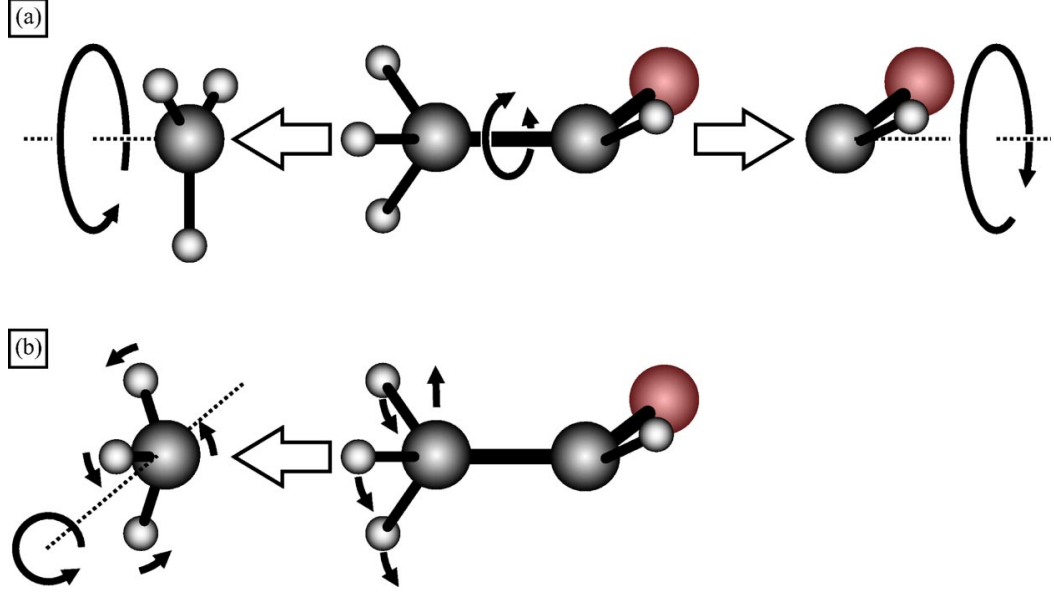

tribution on the image, and therefore the effective rotational temperature was not well defined for the higher vibrational levels.

At the highest photolysis energies studied here, four vibrational bands were observed in the kinetic-energy profile (as shown in Fig. 12). The highest kinetic-energy peak corresponds to ground state $\mathrm{CH}_{3}$, while the second peak, which is only partially resolved, can be confidently assigned to one quanta of excitation in the $\nu_{2}$ umbrella mode. However, the third peak potentially encompasses contributions from more than one vibrational state since the energies of the $(0,2,0,0)$ and $(0,0,0,1)$ levels cannot be resolved with the current instrumental resolution. [The notation $\left(v_{1}, v_{2}, v_{3}, v_{4}\right)$ indicates the number of quanta excited in each vibrational mode, with $\nu_{4}$ denoting the degenerate deformation mode.] Similarly, the $(0,3,0,0)$ and $(0,1,0,1)$ levels may both contribute to the

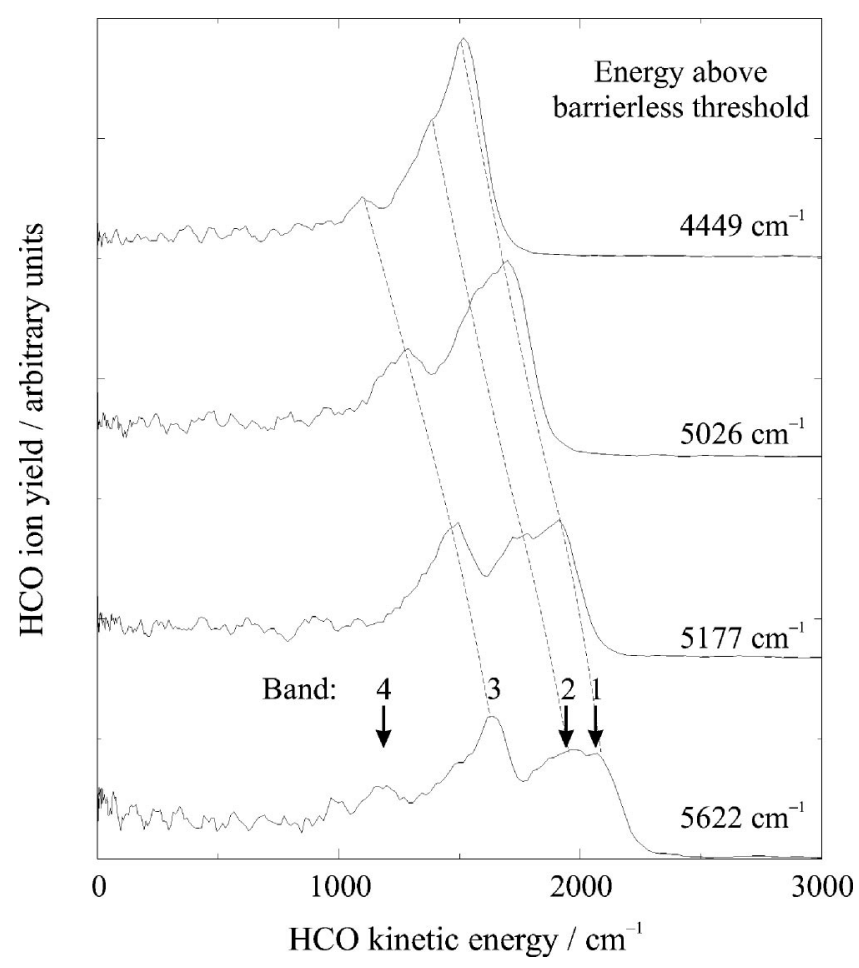

FIG. 12. Kinetic-energy distributions for the HCO photofragment, recorded at a range of photodissociation energies, revealing vibrational excitation of the $\mathrm{CH}_{3}$ coproduct. The identity of bands $1-4$ is discussed in the text.
FIG. 11. (a) Conversion of the $\nu_{15}$ torsional mode of acetaldehyde into rotational excitation of the $\mathrm{CH}_{3}$ and $\mathrm{HCO}$ fragments. (b) Conversion of the $\nu_{14}$ methylrocking mode of $\mathrm{CH}_{3} \mathrm{CHO}$ into tumbling motion of the $\mathrm{CH}_{3}$ product. fourth peak. These composite bands were simulated by assigning all the peak intensity to the $\nu_{2}$ vibrational mode, although the simulations, and thus the integrated intensity of the bands, were relatively insensitive to the vibrational mode chosen. Table IV displays vibrational distributions derived from experimental data collected at a range of photodissociation wavelengths. Each set of data was obtained by averaging over typically two or three images.

The quantitative information that can be extracted from these data is limited by the inconclusive assignments of bands 3 and 4 . Nevertheless, these results provide information that can be used to test the theoretical approaches previously employed to explain product distributions for the $\mathrm{CH}_{3} \mathrm{CHO}$ dissociation. It is of interest to note that the onset of excitation of the lowest vibrational level $v_{2}=1$ does not occur until an excess energy of $\sim 3000 \mathrm{~cm}^{-1}$ (with respect to the barrierless threshold) is reached. However, the fundamental frequency of this mode is $606 \mathrm{~cm}^{-1}$, thus indicating that only a small fraction of the energy released on crossing the barrier is available for partitioning into vibrational excitation of $\mathrm{CH}_{3}$.

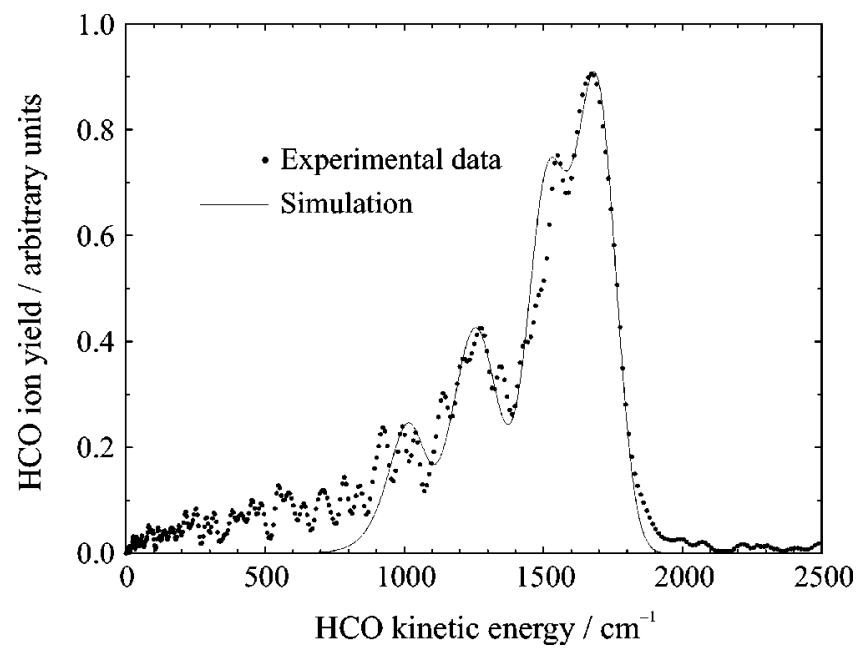

FIG. 13. Simulated and experimental HCO kinetic-energy-release curves corresponding to photodissociation of $\mathrm{CH}_{3} \mathrm{CHO} 5156 \mathrm{~cm}^{-1}$ above the barrierless threshold. The peaks in the distribution are an indication of vibrational excitation of the $\mathrm{CH}_{3}$ cofragment. 
TABLE IV. Vibrational excitation of the $\mathrm{CH}_{3}$ fragment. Available energy is measured with respect to the ground state products and the identity of bands $1-4$ is discussed in the text.

\begin{tabular}{cccccc}
\hline \hline \multirow{2}{*}{$\begin{array}{c}\text { Excitation } \\
\text { energy } \\
\left(\mathrm{cm}^{-1}\right)\end{array}$} & $\begin{array}{c}\text { Available } \\
\text { energy } \\
\left(\mathrm{cm}^{-1}\right)\end{array}$ & Band 1 & Band 2 & Bntensity relative to band 1 \\
\cline { 3 - 6 } & 3529 & 1.00 & 0.23 & 0.12 & 0.27 \\
32619 & 4928 & 1.00 & 0.31 & 0.47 & 0.00 \\
33670 & 5156 & 1.00 & 0.49 & 0.79 & 0.21 \\
34246 & 5753 & 1.00 & 0.56 & 1.12 & 0.19 \\
34843 & 6307 & 1.00 & 0.92 & 0.56 \\
35398 & & &
\end{tabular}

\section{Discussion of vibrational distributions}

In the only previous study of the vibrational excitation of a photodissociation product of $\mathrm{CH}_{3} \mathrm{CHO}$, Hanazaki and co-workers ${ }^{25}$ measured the populations of the lowest $\mathrm{HCO}$ bending and $\mathrm{CO}$-stretch levels over a range of photodissociation wavelengths. The vibrational populations were successfully modeled using a surprisal calculation. ${ }^{6}$ In applying this model, Hanazaki and coworkers split the excess energy into two contributions; the energy released on crossing the barrier, which is mainly partitioned into rotation and translation, and the energy above the barrier, which is distributed statistically over all accessible vibrational modes. In the statistical limit the probability distribution at an available energy $E$ for the vibrational levels of $\mathrm{CH}_{3}$ is given by

$$
P\left(E, v_{i}\right)=\frac{\rho\left(E, v_{i}\right)}{\sum_{v_{i}} \rho\left(E, v_{i}\right)},
$$

where $\rho\left(E, v_{i}\right)$ is the density of states corresponding to the subset of population with $v$ quanta in the $i$ th vibrational mode of $\mathrm{CH}_{3}$. In the present work the $\mathrm{HCO}$ fragment is state-selectively detected and therefore $\rho\left(E, v_{i}\right)$ is simply determined by the translational density of states, which is proportional to $E_{\mathrm{T}}^{1 / 2}$, where $E_{\mathrm{T}}$ is the translational energy. ${ }^{28}$ Table $\mathrm{V}$ displays the experimentally determined vibrational distributions obtained at an excitation energy of $34014 \mathrm{~cm}^{-1}$, $4923 \mathrm{~cm}^{-1}$ above the barrierless threshold, and the corresponding statistical distribution calculated from the translational density of states; it is assumed that the reverse barrier height is $2006 \mathrm{~cm}^{-1}$ (Refs. 4, 17, and 18) and that this energy is partitioned into rotation and translation. An energy of

TABLE V. The vibrational excitation of $\mathrm{CH}_{3}$ calculated according to the statistical model. The experimental data were recorded at a photolysis energy of $34014 \mathrm{~cm}^{-1}$, which is $4923 \mathrm{~cm}^{-1}$ above the barrierless threshold. The height of the reverse barrier on the $T_{1}$ surface is assumed to be $2006 \mathrm{~cm}^{-1}$ (Refs. 4, 17, and 18) and consequently an energy of $2917 \mathrm{~cm}^{-1}$ is available to be statistically distributed across all accessible product states. The intensities of bands 3 and 4 were calculated by summing the contributions of the constituent $\nu_{2}$ and $\nu_{4}$ states.

\begin{tabular}{ccc}
\hline \hline & $\begin{array}{c}\text { Experimental } \\
\text { data }\end{array}$ & $\begin{array}{c}\text { Statistical } \\
\text { prediction }\end{array}$ \\
\hline Band 1 & 1.00 & 1.00 \\
Band 2 & 0.31 & 0.88 \\
Band 3 & 0.27 & 2.18 \\
Band 4 & 0.00 & 1.64 \\
\hline \hline
\end{tabular}

$2917 \mathrm{~cm}^{-1}$ remains to be statistically distributed across the accessible vibrational product states. It is clear that there is much less vibrational excitation of the $\mathrm{CH}_{3}$ fragment than predicted by this surprisal calculation and that the experimental results are therefore not consistent with the simple statistical model.

Terentis et al. have suggested that a low degree of vibrational excitation would be consistent with the impulsive model. ${ }^{2}$ The barrier to dissociation on the $T_{1}$ surface occurs late, at a $\mathrm{C}-\mathrm{C}$ bond length of $2.13 \AA$ (cf. the $1.508 \AA$ bond length in the equilibrium $T_{1}$ geometry), and at this distance the $\mathrm{CH}_{3}$ and $\mathrm{HCO}$ moieties have geometries close to their radical structures. The late barrier inhibits coupling of the available energy into vibrational degrees of freedom and therefore, using the impulsive model, little product vibrational excitation is predicted from consideration of the transition state structure. However, preferential excitation of the $\nu_{2}$ umbrella mode is expected as a result of the "flattening" of the methyl group on dissociation.

If it is assumed, in accordance with the above arguments, that all the observed vibrational excitation is partitioned solely into the $\mathrm{CH}_{3} \nu_{2}$ mode then, as shown in Table IV, it appears that at excitation energies greater than $34500 \mathrm{~cm}^{-1}$ the $v_{2}=2$ level is more highly populated than the $v_{2}=1$ level. This can be explained if it is assumed that there is a significant transfer of vibrational excitation of the methyl group of the parent (in the $S_{1}$ state) into the vibrational modes of the $\mathrm{CH}_{3}$ fragments. The fundamental frequency of the methyl $\nu_{2}$ umbrella mode is $606 \mathrm{~cm}^{-1}$, whereas the corresponding hindered parent mode $\nu_{7}$ has a frequency of $1352 \mathrm{~cm}^{-1}{ }^{29}$ as a direct result of the barrier to inversion. Classically, the preferential population of the $v_{2}=2$ level could be viewed as a consequence of the matching of the parent vibrational frequency with two quanta of excitation in $\nu_{2}$. The quantum picture would describe this in terms of strong FranckCondon overlap between the parent $v_{7}=1$ wave function and the $v_{2}=2$ methyl wave function.

An alternative explanation for the apparent high intensity of the third vibrational band is that it could contain a contribution from excitation of the methyl $\nu_{4}$ mode. No excitation of this mode is predicted from a consideration of the transition state structure, and the methyl deformation modes of acetaldehyde are not among the strongly Franck-Condon active modes for the $S_{0} \rightarrow S_{1}$ excitation. However, IVR could lead to some activity and Baba et al. ${ }^{21}$ saw some evidence of $\mathrm{CH}_{3}$ deformation in the vibronic analysis of the LIF spectrum of acetaldehyde. 

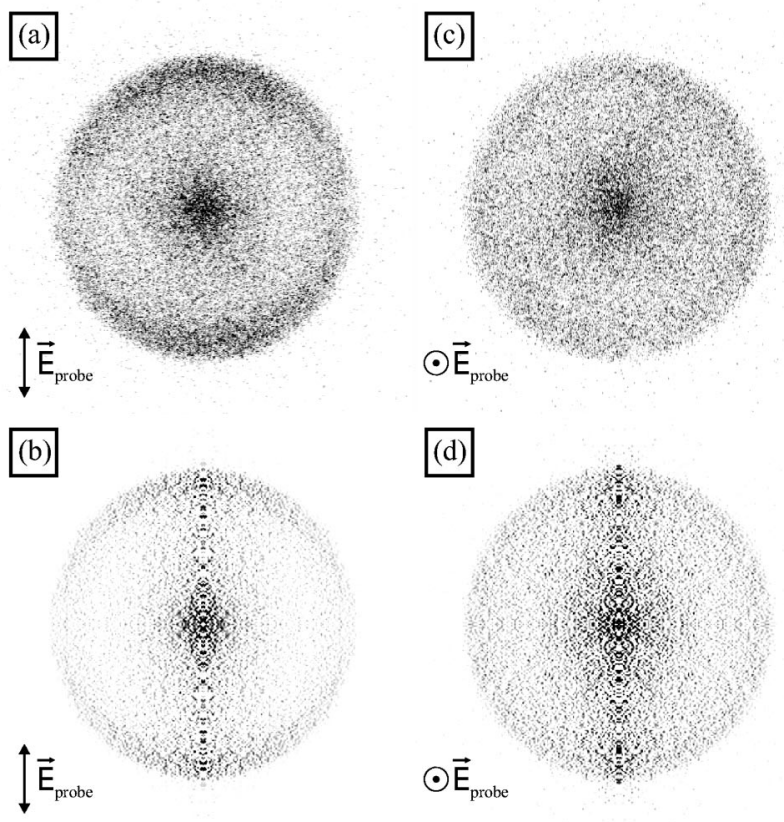

FIG. 14. (a) and (c) show raw ion images of the $\mathrm{CH}_{3}$ fragment obtained with the probe laser polarization parallel and perpendicular to the image plane, respectively. (b) and (d) show the corresponding Abel-inverted images. Images were recorded at an excitation energy of $32619 \mathrm{~cm}^{-1}, 3528 \mathrm{~cm}^{-1}$ above the barrierless threshold for the photodissociation process, and were acquired for 11000 laser shots.

Further experimental data are required in order for these theories to be tested. However, given the current resolution of imaging techniques, it is unrealistic to expect that the contributions of the $\nu_{2}$ and $\nu_{4}$ modes will be resolved via imaging methods in the near future. Therefore, spectroscopic data are needed to determine whether there is significant excitation of the $\nu_{4}$ mode in methyl fragments formed from the photodissociation of $\mathrm{CH}_{3} \mathrm{CHO}$.

\section{VI. $\mathrm{CH}_{3}$ alignment}

\section{A. Anisotropy of $\mathrm{CH}_{3}$ images}

A typical raw image of the $\mathrm{CH}_{3}$ fragment, recorded at a photolysis energy $3528 \mathrm{~cm}^{-1}$ above the barrierless threshold for the formation of ground state $\mathrm{CH}_{3}+\mathrm{HCO}$, is displayed in Fig. 14(a), and in Fig. 14(b) the central slice through the three-dimensional photofragment distribution, reconstructed from the raw image using the BASEX Abel inversion routine, ${ }^{10}$ is shown. As before, $\mathrm{CH}_{3}$ was probed in a $(2+1)$ REMPI scheme via the $Q$ branch of the $3 p\left({ }^{2} A_{2}^{\prime \prime}\right) \leftarrow X\left({ }^{2} A_{2}^{\prime \prime}\right)$ origin band. The observed anisotropy cannot be a manifestation of a $\boldsymbol{\mu}-\mathbf{v}$ correlation since the dissociation process is slow with respect to the rotation of the parent molecule, and there is no anisotropy in the HCO ion images. Instead the anisotropy arises from a correlation between the recoil velocity vector and the angular momentum vector of the methyl fragment ( $\mathbf{v}-\mathbf{J}$ correlation), which is established at the time of fragmentation as a consequence of torques in the transition state and/or conservation of internal rotation and bending motion of the parent. The two-photon excitation probability varies with the angle between the probe laser polarization and the recoil axis, and hence the image is anisotropic. In accordance with this, the anisotropy is lost when the probe laser polarization is rotated through $90^{\circ}$ so as to be perpendicular to the plane of the detector, as indicated in Figs. 14(c) and 14(d).

In order to determine the alignment moments characterizing the anisotropy it would be necessary to obtain a series of images for different rotational transitions of the $\mathrm{CH}_{3}$ excitation spectrum. However, in practice it was only possible to obtain good quality $\mathrm{CH}_{3}$ images by simultaneous excitation of many overlapping $Q$-branch lines, and therefore insufficient experimental data are available to derive these moments. Therefore, a limiting model for the photodissociation process is assumed, as described below, and a forward simulation of the angular distribution is performed by summing the contributions of all accessible $Q$ branch lines. The agreement between experiment and theory confirms that the dynamics are close to the limiting model.

\section{B. Analysis of the $\mathrm{CH}_{3}$ rotational alignment}

In the following analysis, it is assumed that the distribution of $\mathrm{CH}_{3}$ fragment recoil directions is completely isotropic, in view of the slow photodissociation of acetaldehyde. It is also assumed that the alignment of the methyl fragment rotation with respect to the recoil velocity vector is independent of the recoil direction, and is cylindrically symmetrical. Thus it is convenient to define the $z$ axis to lie along the velocity recoil direction, and to characterize the alignment in terms of the distribution of populated $M$ states (the projections of $\mathbf{N}$ quantized along the recoil axis), parametrized using the nonzero alignment moments, ${ }^{30}$

$$
\begin{aligned}
A_{0}^{(0)}= & 1, \\
A_{0}^{(2)}= & \left\langle\left(N, M\left|\left(3 N_{z}^{2}-\mathbf{N}^{2}\right) / \mathbf{N}^{2}\right| N, M\right)\right\rangle, \\
A_{0}^{(4)}= & \left\langle\left( N, M \mid\left(35 N_{z}^{4}-30 N_{z}^{2} \mathbf{N}^{2}+3 \mathbf{N}^{4}+25 N_{z}^{2}\right.\right.\right. \\
& \left.\left.\left.-6 \mathbf{N}^{2}\right) / 8 \mathbf{N}^{4} \mid N, M\right)\right\rangle .
\end{aligned}
$$

As discussed in Sec. V A 3, the main sources of the angular momentum in the $\mathrm{CH}_{3}$ fragment are likely to be the parent internal rotation (the $\nu_{15}$ torsional mode) and the CCO bending vibration (the $\nu_{14}$ methyl-rocking mode). The torsional mode motion of the parent transforms into a propellerlike motion of the $\mathrm{CH}_{3}$ fragment, in which the $\mathbf{N}$ vector points along the recoil direction and the $\mathrm{CH}_{3}$ symmetry axis is also pointing predominantly in that direction: this corresponds to a propensity for forming high- $K$, high- $M$ states [see Fig. 11(a)]. On the other hand, the methyl-rocking motion correlates with a tumbling motion of the $\mathrm{CH}_{3}$ group in which there is little rotation about the symmetry axis (low $K$ ) and the $\mathbf{N}$ vector is primarily perpendicular to the recoil direction [low $M$, see Fig. 11(b)]. There are no parent bending modes that could give rise to the low- $M$, high- $K$ frisbee-like/ cartwheel-like motion, nor to the high- $M$, low- $K$ states, as the $\mathrm{CH}_{3}$ symmetry axis is always perpendicular to the recoil direction in these cases (in contrast to the two $M \sim K$ cases).

Thus, we assume that the projections along the recoil 
direction ( $z$ axis) and the body-fixed axis are highly correlated, i.e., $M \sim K$, and that the expectation values of $M^{2}$ and
$M^{4}$ will be equal to $K^{2}$ and $K^{4}$, respectively. In this limiting case the alignment moments take the values given by ${ }^{31}$

$$
\begin{aligned}
& A_{0}^{(2)}=\frac{3 K^{2}-N(N+1)}{N(N+1)}, \\
& A_{0}^{(4)}=\frac{35 K^{4}-30 K^{2} N(N+1)+3 N^{2}(N+1)^{2}+25 K^{2}-6 N(N+1)}{8 N^{2}(N+1)^{2}} .
\end{aligned}
$$

The populations $P(N, K, M)$ are related to the alignment moments $A_{q}^{(k)}$ by Eq. (19) of Ref. 30,

$$
\begin{aligned}
P(N, K, M)= & n(N, K) \sum_{k q} \xi(N, k) A_{q}^{(k)}(N) \\
& \times \operatorname{Re}\left[\left(N M\left|N_{q}^{(k)}\right| N M\right)\right],
\end{aligned}
$$

where $n(N, K)$ is the total population of level $(N, K)$ summed over all $M$ states, and the values of $\xi(N, k)$ and the matrix elements of $N_{q}^{(k)}$ are calculated as in Ref. 30.

For consistency with our previous analysis we have used a Boltzmann distribution to describe the population of the $(N, K)$ states of $\mathrm{CH}_{3}$. Since for a given $N$ the high- $K$ states lie lower in energy than the low- $K$ states, this will mean that in our model there is a greater propensity assumed for the propeller motion derived from $\nu_{15}$ of the parent than for the tumbling motion derived from $\nu_{14}$.

\section{Simulation of the angular distribution}

At an angle $\theta$ between the recoil direction and the polarization vector, the REMPI signal intensity originating from an individual transition of the $Q$ branch, $I(\theta)$, is given by a sum of partial photoexcitation cross sections,

$$
I(\theta)=\sum_{M^{\prime \prime}} P\left(N^{\prime \prime}, K^{\prime \prime}, M^{\prime \prime}\right) \sigma\left(N^{\prime \prime}, K^{\prime \prime}, M^{\prime \prime} \rightarrow N^{\prime}, K^{\prime}, M^{\prime} ; \theta\right),
$$

where $P\left(N^{\prime \prime}, K^{\prime \prime}, M^{\prime \prime}\right)$ is the population of the particular $M$ level of the ground state, given by Eq. (11) of this paper. To obtain the total ion intensity at a given ejection angle, $I(\theta)$ is summed over all $Q$ branch transitions within the laser bandwidth. The two-photon transition intensity $\sigma$ for excitation of a symmetric top from an initial state $\left\langle N^{\prime \prime} K^{\prime \prime} M^{\prime \prime}\right|$ to a final state $\left|N^{\prime} K^{\prime} M^{\prime}\right\rangle$ can be expressed as

$$
\begin{aligned}
\sigma \propto & \mid \sum_{v} \sum_{\mu_{A} \mu_{B}}\left\langle N^{\prime \prime} K^{\prime \prime} M^{\prime \prime}\left|\mathcal{D}_{A}^{*} \cdot \mathcal{D}_{B}^{*}\right| N^{\prime} K^{\prime} M^{\prime}\right\rangle \\
& \times\left.\left(E_{v i}-\epsilon\right)^{-1} \mu_{f v}^{A} \mu_{v i}^{B}\right|^{2},
\end{aligned}
$$

where

$$
\mathcal{D}_{A}^{*}=\mathcal{D}_{0 \mu_{A}}^{1^{*}} \cos \theta+\mathcal{D}_{1 \mu_{A}}^{1^{*}} \sin \theta-\mathcal{D}_{-1 \mu_{A}}^{1^{*}} \sin \theta .
$$

(Note that for $\theta=0, \mathcal{D}_{A}^{*}=\mathcal{D}_{0 \mu_{A}}^{1^{*}}$, which is the commonly used form for calculating two-photon transition probabilities. ${ }^{32}$ )
In Eqs. (13) and (14), $i, f$, and $v$ denote the ground state, excited state and virtual intermediate state, respectively, $\mu_{A}$ and $\mu_{B}$ represent the projection onto the symmetric-top axis of the angular momenta of photons $A$ and $B$, and take values of $+1,0$, and $-1, \epsilon$ is the energy of the probe photon, $E_{v i}$ is the energy difference between the initial state and the virtual intermediate state, and $\mu_{f v}^{A}$ and $\mu_{v i}^{B}$ represent the vibronic transition moments. The sum over intermediate states $v$ must include all states of appropriate symmetry.

Inserting Eq. (14) into Eq. (13) and using standard angular momentum algebra, the transition probability can be reorganized into the following form:

$$
\begin{aligned}
\sigma \propto & \mid \sum_{\mathcal{Q}=0,2} F(\mathcal{Q})(2 \mathcal{Q}+1)\left[\left(2 N^{\prime \prime}+1\right)\left(2 N^{\prime}+1\right)\right]^{1 / 2} \\
& \times \sum_{\mu_{0} \mu_{0}^{\prime} U_{0} U}\left(\begin{array}{ccc}
1 & 1 & \mathcal{Q} \\
\mu_{0} & \mu_{0}^{\prime} & U_{0}
\end{array}\right)\left(\begin{array}{ccc}
N^{\prime} & \mathcal{Q} & N^{\prime \prime} \\
-M^{\prime} & -U_{0} & M^{\prime \prime}
\end{array}\right) \\
& \times\left(\begin{array}{ccc}
N^{\prime} & \mathcal{Q} & N^{\prime \prime} \\
-K^{\prime} & -U & K^{\prime \prime}
\end{array}\right) \times f^{\mu_{0}}(\theta) f^{\mu_{0}^{\prime}}(\theta) \\
& \times\left.\sum_{\mu_{A} \mu_{B}}(-1)^{\mu_{A}+\mu_{B}-2 \mu_{0}+M^{\prime}-K^{\prime}+U_{0}-U}\left(\begin{array}{ccc}
1 & 1 & \mathcal{Q} \\
\mu_{A} & \mu_{B} & U
\end{array}\right)\right|^{2},
\end{aligned}
$$

where $\mu_{0}$ and $\mu_{0}^{\prime}$ represent the projection of the angular momenta of photons $A$ and $B$ on to the $z$ axis, and can take values of $+1,0$, and $-1, f^{\mu_{0}}(\theta)$ takes the values $\cos \theta$, $\sin \theta$, and $-\sin \theta$ for $\mu_{0}=0,1$, and $-1, U_{0}$ and $U$ denote the projection of $\mathcal{Q}$ on to the $z$ axis and the $\mathrm{CH}_{3}$ symmetric-top axis, respectively, and $\mathcal{Q}$ gives the possible angular momenta arising from the coupling of the two incident photons $A$ and $B$, and takes the values 0 or 2 . Furthermore, the symmetry of the last $3 j$ symbol implies that the $\mathcal{Q}=0$ term can only contribute to the $Q$ branch intensity, and not to $\Delta N \geqslant 1$ transitions. $F(\mathcal{Q})$ is a weighted linear combination of the vibronic transition moments given by 


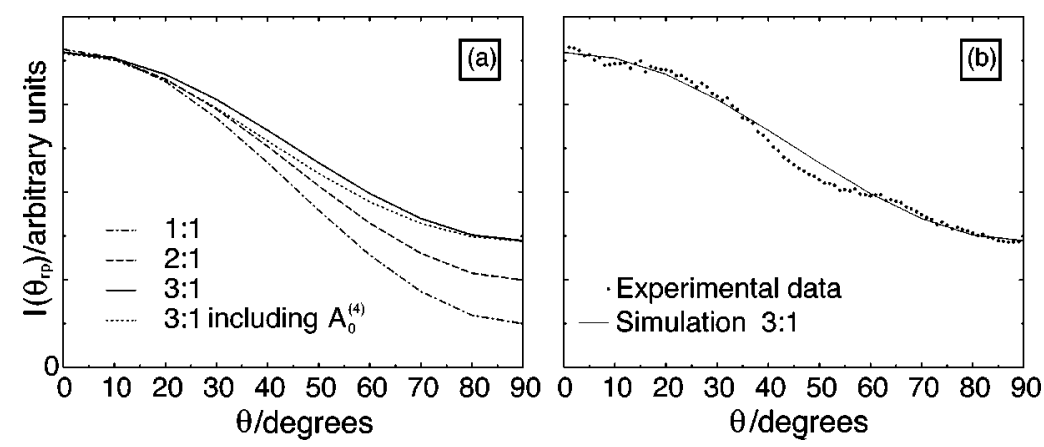

FIG. 15. (a) Simulations of the $\mathrm{CH}_{3}$ angular distribution calculated using the limiting $K \sim M$ model described in the text and assuming different values for the ratio of the $F(\mathcal{Q}=0)$ and $F(\mathcal{Q}=2)$ two-photon excitation parameters. (b) Comparison between the experimental data derived from the reconstructed ion image shown in Fig. 14(b) and the simulation using the $-3: 1$ ratio of $F(\mathcal{Q}=0)$ to $F(\mathcal{Q}=2)$.

$$
F(\mathcal{Q})=\frac{\sum_{\mu_{A} \mu_{B}}\left(\begin{array}{ccc}
1 & 1 & \mathcal{Q} \\
\mu_{A} & \mu_{B} & 0
\end{array}\right) \sum_{v} \frac{\mu_{f v}^{A} \mu_{v i}^{B}}{E_{v i}-\epsilon}}{\sum_{\mu_{A} \mu_{B}}\left(\begin{array}{ccc}
1 & 1 & \mathcal{Q} \\
\mu_{A} & \mu_{B} & 0
\end{array}\right)} .
$$

The values of all quantities in Eqs. (13)-(16) are known, except for the energy differences and the vibronic matrix elements $\mu_{f v}^{A}$ and $\mu_{v i}^{B}$, which contribute to the $F(\mathcal{Q})$ parameters. The ratio of $F(\mathcal{Q}=0)$ to $F(\mathcal{Q}=2)$ was treated as an adjustable parameter in the simulations described here.

The effect of the $F(\mathcal{Q}=0)$ to $F(\mathcal{Q}=2)$ ratio on the calculated angular distribution is shown in Fig. 15(a). The contribution of the $A_{0}^{(4)}$ moment was neglected since it was found to have only a small effect on the simulation. Figure 15(b) displays the best fit with the experimental angular distribution calculated from the reconstructed ion image shown in Fig. 14(b). A $F(\mathcal{Q}=0)$ to $F(\mathcal{Q}=2)$ ratio of $-3: 1$ was utilized, which is consistent with the value obtained by the analysis of the $\mathrm{CH}_{3}$ REMPI spectrum. ${ }^{33}$ However, analysis of spectroscopic data yields only the relative magnitude of the two terms whereas the data presented here allow a derivation of the relative polarity of these vibronic matrix element terms.

The simulation described above neglects effects such as spin depolarization, and therefore the ratio of the $\mathrm{F}(\mathcal{Q}=0)$ to $\mathrm{F}(\mathcal{Q}=2)$ parameters obtained by this method should only be treated as an estimation of the true value. However, the excellent agreement between the experimental and simulated angular distributions is an indication of the validity of the original assumption that the methyl fragment is formed in states with $M \sim K$, and thus the anisotropy is characterized by the limiting values of the alignment moments. This supports the conclusion drawn from the analysis of the product rotational distributions, namely, that the principal source of methyl rotational excitation is the conversion of the acetaldehyde torsional and methyl-rocking vibrations into fragment rotation. A very similar conclusion was drawn by Aoiz and co-workers ${ }^{34}$ in their analysis (closely related to that described in the present work) of $\mathrm{CD}_{3}$ fragment $(\mathbf{v}, \mathbf{J})$ vector correlations in the near $\mathrm{UV}$ photodissociation of $\mathrm{CD}_{3} \mathrm{SCD}_{3}$.

It is important to ask why no alignment of the $\mathrm{HCO}$ fragment is observed. The rotational excitation of the HCO fragment arises from two sources: torque imparted in the exit channel and, in common with $\mathrm{CH}_{3}$, the torsional and bending vibrational modes of acetaldehyde. The torsional mode induces rotational motion about two orthogonal axes, as de- picted in Fig. 11(a), and any alignment of the HCO fragment with respect to its velocity vector is lost in the ensuing tumbling motion.

\section{CONCLUSIONS}

The analysis of rotational and vibrational energy distributions of $\mathrm{HCO}$ and $\mathrm{CH}_{3}$ radicals formed by the photodissociation of $\mathrm{CH}_{3} \mathrm{CHO}$ has given valuable insight into the dynamics of this fragmentation process. The $\mathrm{HCO}$ fragment is formed with greater rotational energy than its $\mathrm{CH}_{3}$ counterpart, which is broadly consistent with an impulsive model of the dissociation process in which the rotational excitation of the products is predicted by conservation of angular momentum. It is proposed that the rotational excitation of $\mathrm{CH}_{3}$ arises from the conversion of the $\mathrm{CH}_{3} \mathrm{CHO}$ torsional and methyl-rocking vibrational modes into rotation on dissociation. This view is consolidated by the observation of rotational alignment in the methyl images, indicating preferential excitation of the high- $K$, high- $M$ propeller motion and low$K$, low- $M$ tumbling motions of $\mathrm{CH}_{3}$. No alignment of the $\mathrm{HCO}$ fragment is observed since exit-channel forces and the torsional excitation of $\mathrm{CH}_{3} \mathrm{CHO}$ induce rotation about orthogonal axes, resulting in tumbling motion.

The vibrational distribution of $\mathrm{CH}_{3}$ reveals preferential excitation of the $\nu_{2}$ umbrella mode, as predicted from the transition state structure, with the possibility of some excitation of the $\nu_{4}$ deformation mode. The distribution is considerably colder than predicted by the statistical models previously used to describe the vibrational excitation of the $\mathrm{HCO}$ cofragment. The observed threshold for the excitation of one quanta of the bending mode is $\sim 3000 \mathrm{~cm}^{-1}$ above the barrierless threshold for the formation of ground state $\mathrm{HCO}$ and $\mathrm{CH}_{3}$, indicating that the energy released on crossing the barrier is not partitioned into vibrational excitation.

Further spectroscopic data are required to determine the relative degrees of excitation of the $\nu_{2}$ and $\nu_{4}$ modes of $\mathrm{CH}_{3}$. Detailed investigations of the $\mathrm{CH}_{3}$ spectroscopy are generally hindered by the effects of predissociation, but these effects are diminished in the deuterated isotope $\mathrm{CD}_{3}$. A study of the photodissociation of deuterated acetaldehyde would provide complementary data to that presented here and would hopefully resolve some of the questions raised. Furthermore, the detection of $\mathrm{CD}_{3}$ through non- $Q$ branch transitions with well-defined rotational quantum numbers would enable a more detailed analysis of the alignment of the methyl fragment. 


\section{ACKNOWLEDGMENTS}

The authers are grateful to Sarah Brownbill and Ben Clarson for their assistance with these experiments. This work was supported financially by the EPSRC under Portfolio Partnership (Grant No.GR/S71750/01).

${ }^{1}$ T. Kono, M. Takayanagi, T. Nishiya, and I. Hanazaki, Chem. Phys. Lett. 201, 166 (1993).

${ }^{2}$ A. C. Terentis, M. Stone, and S. H. Kable, J. Phys. Chem. 98, 10802 (1994).

${ }^{3}$ S.-H. Lee and I.-C. Chen, J. Chem. Phys. 105, 4597 (1996).

${ }^{4}$ J. S. Yadav and J. D. Goddard, J. Chem. Phys. 84, 2682 (1986).

${ }^{5}$ T. Kono, M. Takayanagi, and I. Hanazaki, J. Phys. Chem. 97, 12793 (1993).

${ }^{6}$ E. Zamir and R. D. Levine, Chem. Phys. 52, 253 (1980).

${ }^{7}$ G. E. Busch and K. R. Wilson, J. Chem. Phys. 56, 3626 (1972).

${ }^{8}$ A. T. J. B. Eppink and D. H. Parker, Rev. Sci. Instrum. 68, 3477 (1997)

${ }^{9}$ J. D. Robinson, R. J. Foltynowicz, K. Prentice, P. Bell, and E. R. Grant, J. Chem. Phys. 116, 8384 (2002).

${ }^{10}$ V. Dribinski, A. Ossadtchi, V. A. Mandelshtam, and H. Reisler, Rev. Sci. Instrum. 73, 2634 (2002).

${ }^{11}$ A. T. J. B. Eppink and D. H. Parker, J. Chem. Phys. 110, 832 (1999).

${ }^{12}$ D. W. Chandler, J. W. Thoman, Jr., M. H. M. Janssen, and D. H. Parker, Chem. Phys. Lett. 156, 151 (1989).

${ }^{13}$ S. C. Wang, Phys. Rev. 34, 243 (1929).

${ }^{14}$ R. B. Lawrance and M. W. P. Strandberg, Phys. Rev. 83, 363 (1951).

${ }^{15}$ X.-M. Song and T. A. Cool, J. Chem. Phys. 96, 8664 (1992).
${ }^{16}$ D. C. Moule and K. H. K. Ng, Can. J. Chem. 63, 1378 (1985).

${ }^{17}$ J. A. Altmann, T. A. M. Doust, and A. D. Osborne, Chem. Phys. Lett. 69, 595 (1980).

${ }^{18}$ E. J. P. Malar and A. K. Chandra, J. Phys. Chem. 91, 5111 (1987).

${ }^{19}$ M. Noble and E. K. C. Lee, J. Chem. Phys. 80, 134 (1984).

${ }^{20}$ M. Noble and E. K. C. Lee, J. Chem. Phys. 81, 1632 (1984).

${ }^{21}$ M. Baba, I. Hanazaki, and U. Nagashima, J. Chem. Phys. 82, 3938 (1985).

${ }^{22}$ M. C. Chuang, M. F. Foltz, and C. B. Moore, J. Chem. Phys. 87, 3855 (1987).

${ }^{23}$ V. Špirko and W. P. Kraemer, J. Mol. Spectrosc. 147, 541 (1991).

${ }^{24}$ R. N. Dixon, Acc. Chem. Res. 24, 16 (1991).

${ }^{25}$ T. Gejo, M. Takayanagi, T. Kono, and I. Hanazaki, Chem. Phys. Lett. 218 , 343 (1994).

${ }^{26}$ K. A. Trentelman, S. H. Kable, D. B. Moss, and P. L. Houston, J. Chem. Phys. 91, 7498 (1989).

${ }^{27}$ Y. Kurosaki and K. Yokoyama, Chem. Phys. Lett. 371, 568 (2003).

${ }^{28}$ D. J. Bogan and D. W. Setser, J. Chem. Phys. 64, 586 (1976).

${ }^{29}$ T. Shimanouchi, Tables of Molecular Vibrational Frequencies (National Bureau of Standards, Washington, DC, 1972), Vol.I.

${ }^{30}$ A. C. Kummel, G. O. Sitz, and R. N. Zare, J. Chem. Phys. 85, 6874 (1986).

${ }^{31}$ M. H. M. Janssen, D. H. Parker, G. O. Sitz, S. Stolte, and D. W. Chandler, J. Phys. Chem. 95, 8007 (1991).

${ }^{32}$ K.-M. Chen and E. S. Yeung, J. Chem. Phys. 69, 43 (1978).

${ }^{33}$ H. Dickinson, D. Phil. thesis, University of Oxford, 2000.

${ }^{34}$ J. Barr, I. Torres, L. Banares, J. E. Verdasco, and F. J. Aoiz, Chem. Phys. Lett. 373, 550 (2003). 